\title{
Physical Training Limb Exertion Control Model under Machine Vision and Application in Physical Education
}

\author{
Chen Chonggao \\ Huali College Guangdong University of Technology, Guangzhou, 511325, China \\ E-mail: chonggao1981@163.com
}

Keywords: machine vision; physical training; control; limb; mechanical model

\begin{abstract}
In order to improve the ability of quantitative analysis of human limb force, according to the physical training guidance of limb transceiver force control research, a physical force control model based on machine vision is proposed. The force model of limbs in the course of human body training is constructed by using the 7-link model. The dynamic mechanics and static mechanics analysis method are combined to model the mechanical model of the limb force, and the force and force parameters of the limb are analyzed. The adaptive optimization of mechanical parameters of limbs is carried out by using the method of small disturbance suppression, and the optimal estimation and identification of mechanical parameters are realized. According to the estimation results of limb force parameters, the driving torque of the left and right leg joints of human lower extremity under various motion modes is solved, and the fuzzy control method is used to design the limb force control model. Sports training is guided by the estimation results of physical exertion parameters. The simulation results show that the model is accurate to estimate the body force parameters and the control quality of the limb force is higher. It has a great guiding effect on improving the effect of sports training.
\end{abstract}

\section{Introduction}

In physical training and human sports, physical explosive force plays a fundamental role in achieving excellent sports results and improving human sports potential. Nowadays, there are many forms of sports, such as track and field sports, basketball, and so on. Football, badminton and so on, need to have a strong body burst, to maintain a good level of competitive state. In order to get twice the result with half the effort in physical training, we must analyze the principle and model of human limb force from the angle of human limb dynamics theory, give full play to the potential of human body movement and improve the training effect. The mechanical control model of limbs is studied, and the limb control of human body training is carried out with the method of estimating mechanical parameters, and the force of human limbs is controlled according to the result of parameter estimation, so as to guide physical training. The related control model has been paid great attention to.

In the process of exertion control of human limb movement training, it is easy to be affected by disturbance factors such as joint activity of human limb, which leads to steady state error and 
vibration in the process of posture positioning. Because of the poor stability and the low precision of estimating mechanical parameters ${ }^{[1]}$, it is necessary to optimize the design of posture control for human limb movement training and improve the quality of control, so as to effectively guide sports training. In the traditional method, there are fuzzy control method, integral control method and so on ${ }^{[2-4]}$. The sensor sensitive element is used to collect and measure the force and force parameters of human limbs accurately. Combined with the corresponding control methods, the optimization of attitude parameters is realized, and the mechanical control ability of human limbs is improved. According to the above design principle, the related literature has carried on the optimization design of the human limb force control, and it has obtained the certain research result. In reference [5], a human limb force control model based on the adaptive robust trajectory tracking recognition is proposed. The adaptive position and pose correction method is used to realize the tracking and identification of human limb force trajectory, which can improve the estimation accuracy and control accuracy of the human limb's generating parameters, but the robustness of the control model is not good. The adaptive mechanical tracking ability of force-generating control is not strong. In reference [6], a mechanical spatial force tracking model based on chaotic particle swarm optimization algorithm is proposed to optimize the posture trajectory of human limbs. The ability of force tracking control is improved, but the stability of the method is not good under the influence of human limb disturbance.

In order to solve the above problems, this paper proposes a limb force control model based on machine vision, which uses the 7-link model to construct the limb force model in the course of human body training, and combines the dynamic mechanics and static mechanics analysis method. The mechanical model of limb force is established and the force and force parameters of limbs are analyzed. The adaptive optimization of mechanical parameters of limbs is carried out by using small disturbance suppression method to realize the optimal estimation and identification of mechanical parameters. The fuzzy control method is used to design the limb force control model. Sports training is guided by the estimation results of physical exertion parameters. Finally, a simulation experiment is carried out to demonstrate the superior performance of this method in improving the ability of generating force control of human body training limbs.

\section{Limb Structure Model and Mechanical Analysis under Human Training Mode}

\subsection{Limb structure model under human training mode}

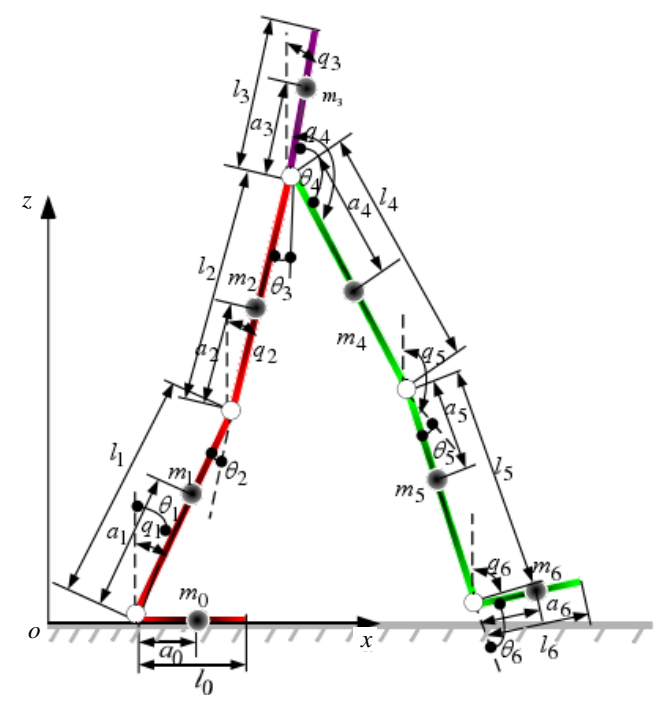

Figure 1 Structure model of limbs with seven links in human training mode 
When the human body sports, mainly by the limb as the force point, it carries on the corresponding sports, needs to carry on the modelling analysis to the human body limb help dynamics, unifies the human body kinematics model to carry on the strength control ${ }^{[7]}$, A 7-link model is used to construct the limb force model during human training, as shown in figure 1.

According to the seven-bar structure model shown in figure 1, the mechanical analysis is carried out. Combined with the results of dynamic analysis ${ }^{[9]}$, the mathematical expression of the dynamic model of human limb booster can be expressed as follows:

$$
M(\theta) \ddot{\theta}+C(\theta, \dot{\theta})+G(\theta)=T_{s}+t_{H}
$$

In this paper, the lower limb running model and the jumping movement model are constructed, and the control analysis of the limb force under the two kinds of motion models is carried out, and the mechanical parameters are estimated, the leg limb is regarded as a whole, the global force is analyzed, and the inertia moment analysis method is combined. By decomposing the seven-link structure of the human body's moving limbs, dynamic mathematical modeling and mechanical analysis are carried out. In the limb motion mode, the limb assisted skeleton can be understood as the 7-link model structure, which is biped and shank, respectively. Seven parts of thigh and upper limb are obtained, and the control constraint parameter model is obtained ${ }^{[8]}$.

\subsection{Construction of mechanical models}

In the world coordinate system Oxyz of the reference configuration distribution of body force object, the coordinate of force point is $\left(x_{a}, 0\right)$, the force center of human limb is $G_{i}\left(x_{i}, z_{i}\right)$, and the center of mass of lower extremity is $G_{i}\left(x_{i}, z_{i}\right)$. The decomposition expression is expressed as follows:

$$
\begin{aligned}
& x_{0}=x_{a}+a \\
& x_{1}=x_{a}+a_{1} \sin q_{1} \\
& x_{2}=x_{a}+l_{1} \sin q_{1}+a_{2} \sin q_{2} \\
& x_{3}=x_{a}+l_{1} \sin q_{1}+l_{2} \sin q_{2}+a_{3} \sin q_{3} \\
& x_{4}=x_{a}+l_{1} \sin q_{1}+l_{2} \sin q_{2}+a_{4} \sin q_{4} \\
& x_{5}=x_{a}+l_{1} \sin q_{1}+l_{2} \sin q_{2}+l_{4} \sin q_{4}+a_{5} \sin q_{5} \\
& x_{6}=x_{a}+l_{1} \sin q_{1}+l_{2} \sin q_{2}+l_{4} \sin q_{4}+l_{5} \sin q_{5}+a_{6} \sin q_{6}
\end{aligned}
$$

For $z_{i}$, the fuzzy reference coefficients of limb return are obtained by combining with dynamic control method, as:

$$
\begin{aligned}
& z_{0}=0 \\
& z_{1}=a_{1} \cos q_{1} \\
& z_{2}=l_{1} \cos q_{1}+a_{2} \cos q_{2} \\
& z_{3}=l_{1} \cos q_{1}+l_{2} \cos q_{2}+a_{3} \cos q_{3} \\
& z_{4}=l_{1} \cos q_{1}+l_{2} \cos q_{2}+a_{4} \cos q_{4} \\
& z_{5}=l_{1} \cos q_{1}+l_{2} \cos q_{2}+l_{4} \cos q_{4}+a_{5} \cos q_{5} \\
& z_{6}=l_{1} \cos q_{1}+l_{2} \cos q_{2}+l_{4} \cos q_{4}+l_{5} \cos q_{5}+a_{6} \cos q_{6}
\end{aligned}
$$


The inertial integral compensation method is used to track the force steady-state, and the inertial potential energy of the body motion is measured as follows:

$$
K=\frac{1}{2} \sum_{i=0}^{6}\left[I_{i} \dot{q}_{i}^{2}+m_{i}\left(\dot{x}_{i}^{2}+\dot{z}_{i}^{2}\right)\right]
$$

In the upper formula, $I_{i}$ is the moment of inertia of each limb in motion around its corresponding center of mass. The potential energy of forward movement of human body in sports is:

$$
P=\sum_{i=0}^{6} m_{i} g z_{i}
$$

Based on the above analysis, the mechanical model of the force on the limbs of human body motion is constructed. On the basis of this, the force and force parameters of the limbs are analyzed, and the optimal design of the force control model is carried out ${ }^{[10]}$.

\section{Control Model Optimization}

\subsection{Optimal estimation and identification of mechanical parameters}

Combined with dynamic mechanics and static mechanics analysis, the mechanical model of limb force is modeled $^{[11]}$. At this time, the Lagrange function of limb structure is:

$$
L=K-P
$$

Thus, the Lagrange kinetic equation of limb assisted motion is obtained as follows:

$$
\frac{d}{d t}\left[\frac{\partial L}{\partial \dot{q}_{i}}\right]-\frac{\partial L}{\partial q_{i}}=T_{i},(i=1,2, \cdots, 6)
$$

In the upper formula, $T_{i}$ is the joint moment of the limb connecting rod, the mass matrix of the limb is represented by $M$, the gravity vector is represented by the vector $G$, and the joint torque under the dynamic model of limb force is obtained as follows:

$$
M(q) \ddot{q}+K(q, \dot{q})+H(q)=T
$$

According to Cartesian space trajectory tracking theory, the adaptive optimization of limb mechanical parameters is carried out by using small disturbance suppression method. The matching mode of trajectory between Cartesian space and joint space is obtained, and the phase of limb motion mode is represented by $\theta_{i}(i=1,2, \cdots, 6)$, an angle of the joint, so as to have:

$$
\left\{\begin{array}{l}
\theta_{1}=q_{1} \\
\theta_{2}=q_{1}-q_{2} \\
\theta_{3}=q_{2}-q_{3} \\
\theta_{4}=q_{4}-q_{3} \\
\theta_{5}=q_{5}-q_{4} \\
\theta_{6}=q_{5}-q_{6}
\end{array}\right.
$$

Combined with the angle transformation model under Cartesian space trajectory matching model, the optimized Lagrange equation is obtained as follows:

$$
D(\theta) \ddot{\theta}+C(\theta, \dot{\theta})+G(\theta)=\tau
$$


Where, $\tau=\left[\tau_{1}, \tau_{2}, \ldots, \tau_{5}\right], \tau_{1}, \tau_{2}$ and $\tau_{3}$ are the torque of the ankle joint, the knee joint and the hip joint, respectively. $\tau_{4}$ and $\tau_{5}$ are the driving force moment of the hip joint exit and the ankle joint of the swing leg, respectively.

\subsection{Fuzzy control of limb force}

According to the estimation results of limb force parameters, the driving moment of the left and right leg joints of the human lower extremity under various motion modes is solved as $x_{i}, \quad i=0,1,2,3$, Thus, the kinetic energy expression of the left leg in running mode is as follows:

$$
K=\frac{1}{2} \sum_{i=0}^{2}\left[I_{i} \dot{q}_{i}^{2}+m_{i}\left(\dot{x}_{i}^{2}+\dot{z}_{i}^{2}\right)+\frac{1}{2}\left(I_{L 3} \dot{q}_{3}^{2}+m_{L 3}\left(\dot{x}_{3}^{2}+\dot{z}_{3}^{2}\right)\right)\right]
$$

Where, $I_{i}$ is the moment of inertia of the member around its center of mass. In the same way, the fuzzy control method is used to design the limb force control model, and the relationship between the adjacent joint nodes' rotation angle in running mode is obtained as follows:

$$
q_{1}=\theta_{1}, \quad q_{2}=\theta_{1}-\theta_{2}, \quad q_{3}=\theta_{1}-\theta_{2}+\theta_{3}
$$

The potential energy of the left leg is expressed as follows:

$$
P=\sum_{i=0}^{2}\left(m_{i} g z_{i}\right)+m_{L 3} g z_{3}
$$

The kinetic energy of the right leg is expressed as:

$$
K=\frac{1}{2}\left(I_{R 3} \dot{q}_{3}^{2}+m_{R 3}\left(\dot{x}_{3}^{2}+\dot{z}_{3}^{2}\right)+\frac{1}{2} \sum_{i=1}^{5}\left[I_{i} \dot{q}_{i}^{2}+m_{i}\left(\dot{x}_{i}^{2}+\dot{z}_{i}^{2}\right)\right]\right.
$$

The motion potential of the right leg is:

$$
P=\sum_{i=1}^{5}\left(m_{i} g z_{i}\right)+m_{R 3} g z_{3}
$$

In addition to the Lagrange dynamic equation, the moment of driving force of each joint of the right leg can be obtained. The equation is:

$$
\frac{d}{d t}\left[\frac{\partial L}{\partial \dot{\theta}_{i}}\right]-\frac{\partial L}{\partial \theta_{i}}=\tau_{i}^{\prime}, \quad i=3,4,5, \quad L=K-P
$$

At this time, the obtained $\tau_{i}^{\prime}$ represents the driving moment of each joint respectively, and the above results are analyzed.

\section{Simulation Experiment and Result Analysis}

On the basis of the above control model and algorithm design, in order to test the application performance of the method in this paper, simulation experiment, system test and data analysis are carried out under machine vision, and ADAMS software is used. A simplified computer vision simulation system for dynamic analysis of limb force in human motion model is obtained. The interface is shown in figure 2. 


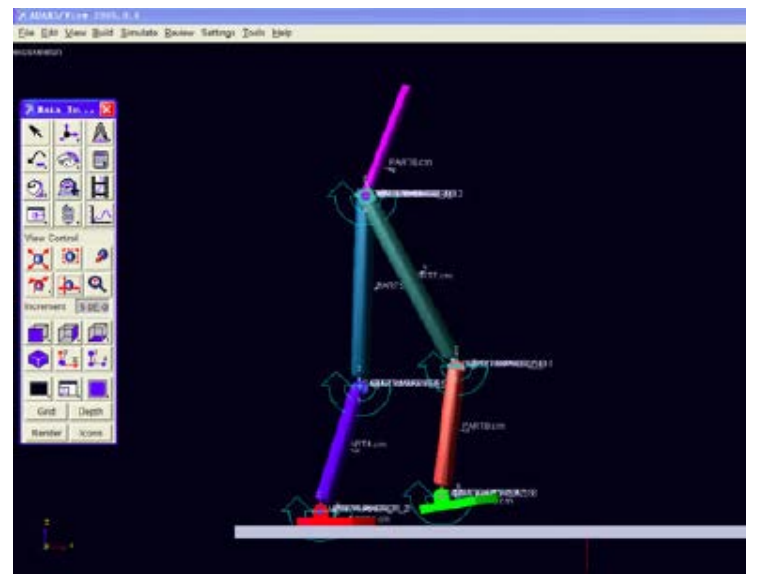

Figure 2 Machine vision simulation interface

Through MATLAB programming, the moment value of each joint is obtained. First, the angle value of joint under experimental condition is given. Then, the analysis data is imported into ADAMS software, and the basic information is set up. In the simulation design, the human motion model limb is first designed. The joints of the body are driven independently to make the joints and limbs run according to the predetermined trajectory mode in the single leg support motion mode and the running motion mode. With the movement of the limbs, the movement data of the joints of the limbs are kept as .txt text data, and the data format is adjusted according to the system information requirements of the ADAMS software, which is consistent with the rhythm of the human body movement, and the movement data of each joint of the limbs are saved as .txt text data under the autonomous drive. The data format is multidimensional, in which the first column represents the time variable, the second column represents the limb joint motion moment variable, the Spline curve is generated in ADMAS, and the ankle joint torque is generated in the motion mode. The simulation results of the torque control curve of the knee joint are shown in figure 3 .

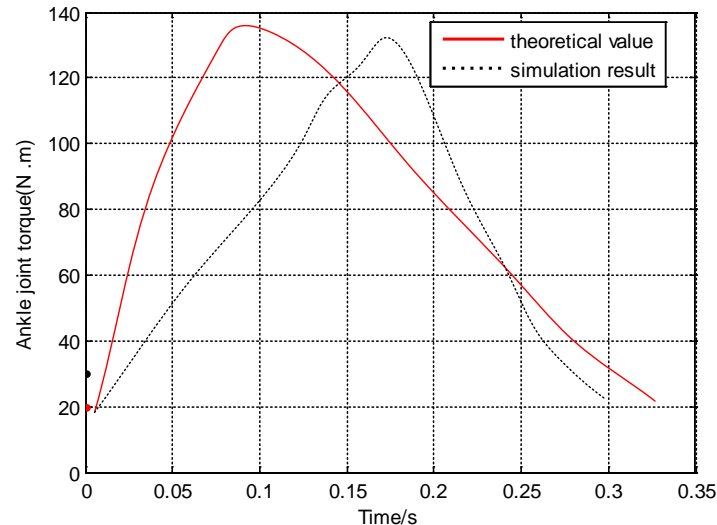

(a) Theoretical and simulation values of ankle joint torque

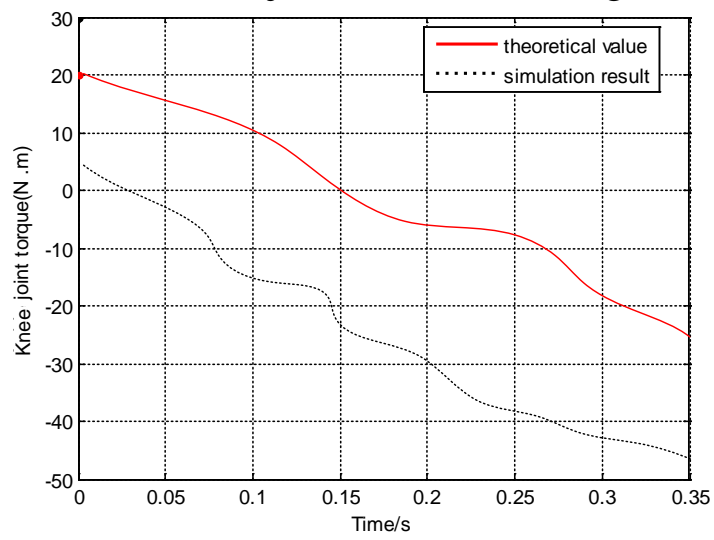

(b) Theoretical and simulation values of knee joint torque

Figure 3 Limb force control performance curve

The simulation results of figure 3 show that the model of this paper is used to control the exertion of limbs trained by human body, and the estimation accuracy of the parameters of limb force is higher, and the control quality of limb force is higher.

\section{Conclusions}

In this paper, a limb force control model based on machine vision is proposed. The force model 
of limbs in the course of human training is constructed by using the 7-link model. Combined with dynamic mechanics and static mechanics analysis, the mechanics of limb force is carried out. The model modeling, the analysis of the force and force parameters of the limbs, the adaptive optimization of the mechanical parameters of the limbs using the small disturbance suppression method, the optimal estimation and identification of the mechanical parameters are realized. According to the estimation results of limb force parameters, the driving torque of the left and right leg joints of human lower extremity under various motion modes is solved. The limb force control model is designed by using fuzzy control method, and the limb force is estimated according to the parameters of limb force. The results guide sports training. The results show that the proposed model has a high accuracy in estimating the body force parameters and has a good application value in sports training instruction.

\section{References}

[1] Guo X, Ma S G, Li B, et al. Velocity tracking control of a snake-like robot with a dynamics and control unified model [J]. Acta Automatica Sinica, 2015, 41(11):1847-1856.

[2] Zhang A F, Ma S G, Li B, et al. Adaptive controller design for underwater snake robot with unmatched uncertainties [J]. Science China: Information Sciences, 2016, 59(5):No.052205.

[3] ZHANG Danfeng, LI Bin, WANG Liyan. Tracking Control Method of the Centre-of-Mass Velocity for aSnake-like Robot Based on the Continuum Model. ROBOT, 2017, 39(6): 829-837.

[4] Varley J, Weisz J, Weiss J, et al. Generating multi-fingered robotic grasps via deep learning[C]//IEEE/RSJ International Conference on Intelligent Robots and Systems. Piscataway, USA: IEEE, 2015:4415-4420.

[5] Girshick R, Donahue J, Darrell T, et al. Rich feature hierarchies for accurate object detection and semantic segmentation[C]//IEEE Conference on Computer Vision and Pattern Recognition. Piscataway, USA: IEEE, 2014: 580-587.

[6] HOU Che, WANG Zheng, ZHAO Yiwen, SONG Guoli. Load Adaptive Force-free Control for the Direct Teaching of Robots. ROBOT, 2017, 39(4): 439-448.

[7] You Y P, Zhang Y, Li C G. Force-free control for the direct teaching of robots [J]. Journal of Mechanical Engineering, 2014, 50(3):10-17.

[8] Uijlings $J R R$, van de Sande K E A, Gevers T, et al. Selective search for object recognition [J]. International Journal of Computer Vision, 2013, 104(2):154-171.

[9] XU Shan, LI Gaofeng, LIU Jingtai, HAO Jie. Inverse Kinematics Solution of Deformable Manipulator for Point Touching Task. ROBOT, 2017, 39(4): 405-414.

[10] Muhammad J, Altun H, Abo-Serie E. Welding seam profiling techniques based on active vision sensing for intelligent robotic welding [J]. International Journal of Advanced Manufacturing Technology, 2016, 88(1-4): 127-145.

[11] Manorathna R P, Phairatt P, Ogun P, et al. Feature extraction and tracking of a weld joint for adaptive robotic welding[C]//13th International Conference on Control, Automation, Robotics \& Vision. Piscataway, USA: IEEE, 2014: 1368-1372. 